\title{
National, regional and global mortality due to alcoholic cardiomyopathy in 2015
}

\author{
Jakob Manthey, ${ }^{1}$ Charlotte Probst, ${ }^{1,2}$ Margaret Rylett, ${ }^{2}$ Jürgen Rehm ${ }^{1,2,3,4,5,6}$
}

- Additional material is

published online only. To view please visit the journal online (http://dx.doi.org/10.1136/ heartjn-2017-312384).

${ }^{1}$ Institute for Clinical Psychology and Psychotherapy, TU Dresden, Dresden, Germany

${ }^{2}$ Institute for Mental Health

Policy Research, Centre for Addiction and Mental Health,

Toronto, Ontario, Canada ${ }^{3}$ Campbell Family Mental Health Research Institute, Centre for Addiction and Mental Health,

Toronto, Ontario, Canada ${ }^{4}$ Institute of Medical Science (IMS), University of Toronto, Toronto, Ontario, Canada ${ }^{5}$ Department of Psychiatry, University of Toronto, Toronto, Ontario, Canada

${ }^{6}$ Dalla Lana School of Public Health, University of Toronto, Toronto, Ontario, Canada

\section{Correspondence to}

Jakob Manthey, Institute for Clinical Psychology and Psychotherapy, TU Dresden, Dresden 01187, Germany; jakob.manthey@tu-dresden.de

Received 4 September 2017 Revised 12 February 2018 Accepted 13 February 2018 Published Online First 13 March 2018

\section{ABSTRACT \\ Objectives (1) A comprehensive mortality assessment of alcoholic cardiomyopathy (ACM) and (2) examination of under-reporting using vital statistics data. \\ Methods A modelling study estimated sex- specific mortality rates for each country, which were subsequently aggregated by region and globally. Input data on ACM mortality were obtained from death registries for $n=91$ countries. For $n=99$ countries, mortality estimates were predicted using aggregate alcohol data from WHO publications. Descriptive additional analyses illustrated the scope of under- reporting.}

Results In 2015, there were an estimated 25997 (95\% Cl 17385 to 49 096) global deaths from ACM. This translates into $6.3 \%(95 \% \mathrm{Cl} 4.2 \%$ to $11.9 \%)$ of all global deaths from cardiomyopathy being caused by alcohol. There were large regional variations with regard to mortality burden. While the majority of ACM deaths were found in Russia (19749 deaths, 76.0\% of all ACM deaths), for about one-third of countries $(n=57)$ less than one ACM death was found. Under-reporting was identified for nearly every second country with civil registration data. Overall, two out of three global ACM deaths might be misclassified.

Conclusions The variation of ACM mortality burden is greater than for other alcohol-attributable diseases, and partly may be the result of stigma and lack of detection. Misclassification of ACM fatalities is a systematic phenomenon, which may be caused by low resources, lacking standards and stigma associated with alcoholuse disorders. Clinical management may be improved by including routine alcohol assessments. This could contribute to decrease misclassifications and to provide the best available treatment for affected patients.

\section{INTRODUCTION}

Cardiomyopathy refers to a heterogeneous group of diseases affecting the heart muscle, ${ }^{1}$ where the heart muscle becomes enlarged, thick or rigid. Cardiomyopathy is one of the major heart disease categories, responsible for about 400000 adult deaths $(15+$ years of age) per year globally and thus for $0.7 \%$ of all adult deaths (women: $0.7 \%$; men $0.8 \%$ ) and $2.2 \%$ of all cardiovascular deaths (women: $2.0 \%$; men: 2.5 ). ${ }^{2}$ Chronic heavy alcohol use is linked to dilated cardiomyopathy by ethanol acting as a toxin to weaken the heart muscle directly (see Rehm et al $^{3}$ for the most recent review; Rubin ${ }^{4}$ and Urbano-Marquez et $a l^{5}$ for early landmark studies; Song and Rubin ${ }^{6}$ for an experimental demonstration of the toxic effect of alcohol). One prominent description of what is now called alcoholic cardiomyopathy (ACM) was by a Munich pathologist, who labelled the phenomenon the 'Münchner Bierherz' (the Munich beer heart) and defined it as a disease characterised by cardiac dilatation and hypertrophy due to heavy consumption of beer over time. ${ }^{7}$ More recent research indicated that ACM is a specific form of dilated cardiomyopathy, caused by both ethanol (pure alcohol) and acetaldehyde, the first metabolite of ethanol, and that it can be worsened by interaction with other toxins, such as heavy metals, or by lack of nutrients. ${ }^{8}$

Civil registration data are lacking for the majority of the world population, ${ }^{9}$ and ACM is too specific a cause that cannot by assessed via verbal autopsies as the alternative way of estimating causes of death. Thus, a systematic assessment of ACM mortality on a global scale requires mortality modelling for countries without available data. ${ }^{10}$ Recently, ACM deaths and related disability have been included in the Global Burden of Disease (GBD) mortality analyses for the first time. ${ }^{11}$ According to the GBD study, ACM has caused 81600 global deaths in 2015. While these estimates provide an approximation of the true mortality burden attributable to ACM, only a proportion of these deaths might actually be recognised as such. As for other alcohol-attributable diseases, ${ }^{12}$ it is likely that ACM is systematically misclassified at death.

This contribution aims (1) to provide a comprehensive epidemiological assessment of ACM mortality and (2) to examine the scope of under-reporting. This study uses mortality data from countries with vital statistics to develop a statistical estimation model based on alcohol exposure. This model estimated the number of ACM deaths registered in 2015 for each country, for all regions and globally. In order to allow for national and regional comparisons, mortality rates (number of ACM deaths per 1000000 adult population) and alcohol-attributable fractions (AAF; the proportion of all deaths from cardiomyopathy attributable to alcohol consumption) were calculated.

\section{METHODS}

The methods used were developed and described in detail elsewhere. ${ }^{10}$ We will restrict ourselves to sketching out the main modelling strategy here. The online supplementary appendix contains more details on data definitions, the analytic strategy and statistical modelling. Further, all data used for modelling purposes can be found in the online supplementary web table. 


\section{Modelling strategy}

Civil registration data on ACM deaths were available for $n=91$ countries. For the remaining countries $(n=99)$, the sex-specific numbers of ACM deaths were estimated based on regression models, using adult alcohol per capita consumption, the prevalence of alcohol use disorder, population size and the number of deaths from cardiomyopathy as predictors. After combining the recorded and estimated ACM mortality data, sex-specific mortality rates and AAFs were calculated based on the ACM death counts for all countries $(n=190)$, regions, and globally.

\section{Additional analyses}

Under-reporting of ACM mortality in vital statistics was examined in two additional analyses. First, ACM mortality data directly available from vital statistics were compared with the model predictions. Second, all ACM mortality data-from vital statistics or from predictions-were compared with the GBD estimates.

\section{Data sources}

ACM mortality data were mainly obtained from the WHO mortality data base. ${ }^{13}$ The covariates were obtained from the Global Information System on Alcohol and Health ${ }^{14}$ (alcohol per capita consumption) or the Global Status Report on Alcohol and Health ${ }^{15}$ (prevalence of alcohol use disorder). For all countries, the number of cardiomyopathy deaths was obtained from the WHO Global Health Estimates database, ${ }^{2}$ and comparative ACM mortality estimates were sourced from the GBD study. ${ }^{11}$ For all variables, data from the year 2015, or the last available year prior to 2015 were used.

\section{RESULTS}

\section{Distribution of ACM deaths}

Globally, 25997 people died from ACM in 2015 (women: 5726; men: 20 272; see table 1 for regional distribution and CIs). The majority of fatalities were heavily concentrated in the Eastern European region, where $80.1 \%$ of all deaths were estimated to occur. In Russia, 19749 ACM deaths were recorded, which accounted for $76.0 \%$ of all global ACM deaths. In contrast, less than one ACM death was found for 57 countries.

With regard to mortality rates, a similar pattern was observed (see table 1). Globally, the mortality rate was estimated at 4.9 deaths per 1000000 adults (women: 2.1; men: 7.6). However, in most regions (18 out of 21 ) and countries (159 out of 190) mortality rates were below the global average. In 110 countries, less than one adult in 1000000 was estimated to die from ACM. Mortality rates above 10 deaths per 1000000 people were observed in 23 countries. Only in Central and Eastern Europe, the average mortality rates exceeded 10 per 1000000 . The largest mortality rates were observed in Russia (163.8 per 1000 000). Figure 1 illustrates the distribution of mortality rates across all countries.

\section{Distribution of AAF}

The regional estimates of AAFs and their CIs are presented in table 2. Globally, $6.3 \%$ of all deaths from cardiomyopathy were attributable to alcohol (women: 3.1\%; men: 9.0\%). The geographical distribution of AAFs was similar to the distribution of mortality rates. In the vast majority of regions (19 out of 21 ) and countries (166 out of 190), AAFs were below the global average. In 103 countries, AAFs were below 1\%. Only in 21

Table 1 Regional and global distribution of deaths due to alcoholic cardiomyopathy and of mortality rates by sex

\begin{tabular}{|c|c|c|c|c|c|c|}
\hline \multirow[b]{2}{*}{ Region } & \multicolumn{3}{|l|}{ Number of ACM deaths } & \multicolumn{3}{|l|}{ Mortality rate } \\
\hline & Total $(95 \% \mathrm{CI})$ & Female $(95 \% \mathrm{CI})$ & Male $(95 \% \mathrm{CI})$ & Total $(95 \% \mathrm{Cl})$ & Female $(95 \% \mathrm{CI})$ & Male $(95 \% \mathrm{CI})$ \\
\hline Andean Latin America & $2(0$ to 17$)$ & $1(0$ to 8$)$ & $1(0$ to 9$)$ & 0.1 (0.0 to 0.5$)$ & $0.1(0.0$ to 0.4$)$ & $0.1(0.0$ to 0.5$)$ \\
\hline Australasia & 73 (42 to 124$)$ & 10 (0 to 31$)$ & 63 (42 to 93) & 3.2 (1.8 to 5.5$)$ & 0.8 (0.0 to 2.7$)$ & 5.6 (3.8 to 8.2 ) \\
\hline Caribbean & 94 (86 to 129$)$ & 6 (4 to 24$)$ & 88 (82 to 104$)$ & 3.4 (3.1 to 4.7$)$ & 0.5 (0.3 to 1.8$)$ & 6.5 (6.1 to 7.8$)$ \\
\hline Central Asia & 323 (300 to 365$)$ & 58 (54 to 66$)$ & 265 (246 to 298 ) & 5.1 (4.8 to 5.8 ) & 1.7 (1.6 to 2.0$)$ & 8.7 (8.1 to 9.8$)$ \\
\hline Central Europe & 1101 (554 to 2874) & 194 (64 to 841) & 907 (522 to 2033) & 11.2 (5.6 to 29.2 ) & $3.8(1.3$ to 16.5$)$ & 19.2 (11.1 to 43.0$)$ \\
\hline Central Latin America & 48 (35 to 89 ) & $3(1$ to 17$)$ & 45 (35 to 72 ) & $0.3(0.2$ to 0.5$)$ & $0.0(0.0$ to 0.2$)$ & 0.5 (0.4 to 0.8$)$ \\
\hline $\begin{array}{l}\text { Central Sub-Saharan } \\
\text { Africa }\end{array}$ & 56 (31 to 104) & 17 (7 to 41$)$ & 39 (24 to 63) & $0.9(0.5$ to 1.7$)$ & $0.5(0.2$ to 1.3$)$ & 1.3 (0.8 to 2.1$)$ \\
\hline East Asia & 242 (104 to 585$)$ & 20 (4 to 94$)$ & 222 (100 to 491$)$ & 0.2 (0.1 to 0.5$)$ & $0.0(0.0$ to 0.2$)$ & $0.4(0.2$ to 0.8$)$ \\
\hline Eastern Europe & 21007 (14972 to 37380$)$ & 5029 (4341 to 8193) & 15978 (10658 to 29472$)$ & 119.8 (85.4 to 213.1 ) & 52.6 (45.4 to 85.7) & 200.2 (133.5 to 365.6$)$ \\
\hline $\begin{array}{l}\text { Eastern Sub-Saharan } \\
\text { Africa }\end{array}$ & 140 (92 to 221$)$ & 19 (9 to 42$)$ & 120 (82 to 179$)$ & 0.7 (0.4 to 1.1$)$ & $0.2(0.1$ to 0.4$)$ & 1.2 (0.8 to 1.8$)$ \\
\hline High-income Asia Pacific & 48 (3 to 166$)$ & 2 (0 to 45$)$ & 46 (11 to 122$)$ & 0.3 (0.0 to 1.1$)$ & 0.0 (0.0 to 0.6$)$ & $0.6(0.2$ to 1.7$)$ \\
\hline $\begin{array}{l}\text { High-income North } \\
\text { America }\end{array}$ & 585 (218 to 1450$)$ & 93 (7 to 374$)$ & 492 (211 to 1076) & 2.0 (0.8 to 5.1$)$ & $0.6(0.1$ to 2.6$)$ & 3.5 (1.5 to 7.6$)$ \\
\hline $\begin{array}{l}\text { North Africa and Middle } \\
\text { East }\end{array}$ & 50 (18 to 166$)$ & 17 (5 to 70$)$ & 33 (13 to 95$)$ & $0.1(0.0$ to 0.4$)$ & $0.1(0.0$ to 0.4$)$ & $0.2(0.1$ to 0.5$)$ \\
\hline Oceania & $3(1$ to 10$)$ & 2 (0 to 7 ) & 1 (0 to 3 ) & $0.5(0.2$ to 1.6$)$ & $0.6(0.2$ to 2.2$)$ & $0.4(0.2$ to 1.1$)$ \\
\hline South Asia & 92 (41 to 213 ) & $6(2$ to 30$)$ & 85 (40 to 184$)$ & 0.1 (0.0 to 0.2$)$ & $0.0(0.0$ to 0.1$)$ & $0.2(0.1$ to 0.3$)$ \\
\hline Southeast Asia & 96 (43 to 234$)$ & 17 (6 to 62$)$ & 79 (39 to 173$)$ & 0.2 (0.1 to 0.5$)$ & $0.1(0.0$ to 0.3$)$ & $0.4(0.1$ to 0.7$)$ \\
\hline Southern Latin America & 23 (5 to 135$)$ & 1 (0 to 41$)$ & 22 (6 to 94$)$ & 0.5 (0.1 to 2.8$)$ & 0.0 (0.0 to 1.6$)$ & $0.9(0.3$ to 4.0$)$ \\
\hline $\begin{array}{l}\text { Southern Sub-Saharan } \\
\text { Africa }\end{array}$ & 179 (108 to 299$)$ & 21 (10 to 44$)$ & 159 (99 to 255 ) & $3.4(2.1$ to 5.7$)$ & $0.8(0.4$ to 1.6$)$ & 6.1 (3.8 to 9.9) \\
\hline Tropical Latin America & 260 (112 to 565$)$ & 20 (2 to 72$)$ & 240 (111 to 493$)$ & $1.6(0.7$ to 3.5$)$ & $0.2(0.0$ to 0.9$)$ & $3.0(1.4$ to 6.3$)$ \\
\hline Western Europe & 974 (251 to 2875$)$ & 145 (13 to 994$)$ & 830 (275 to 1881$)$ & 2.7 (0.7 to 8.1$)$ & 0.8 (0.1 to 5.5$)$ & $4.8(1.6$ to 10.9$)$ \\
\hline $\begin{array}{l}\text { Western Sub-Saharan } \\
\text { Africa }\end{array}$ & 602 (341 to 1094) & 45 (17 to 134$)$ & 556 (325 to 960$)$ & $2.7(1.5$ to 5.0$)$ & 0.4 (0.1 to 1.2$)$ & 5.0 (2.9 to 8.7$)$ \\
\hline Global & 25997 (17358 to 49 096) & 5726 (4548 to 11230$)$ & 20272 (12920 to 38150$)$ & 4.9 (3.2 to 9.2$)$ & 2.1 (1.7 to 4.2 ) & 7.6 (4.8 to 14.2$)$ \\
\hline
\end{tabular}

Mortality rates denote the number of deaths per 1000000 adult population. Definition of regions are based on the Global Burden of Disease study.11

ACM, alcoholic cardiomyopathy. 

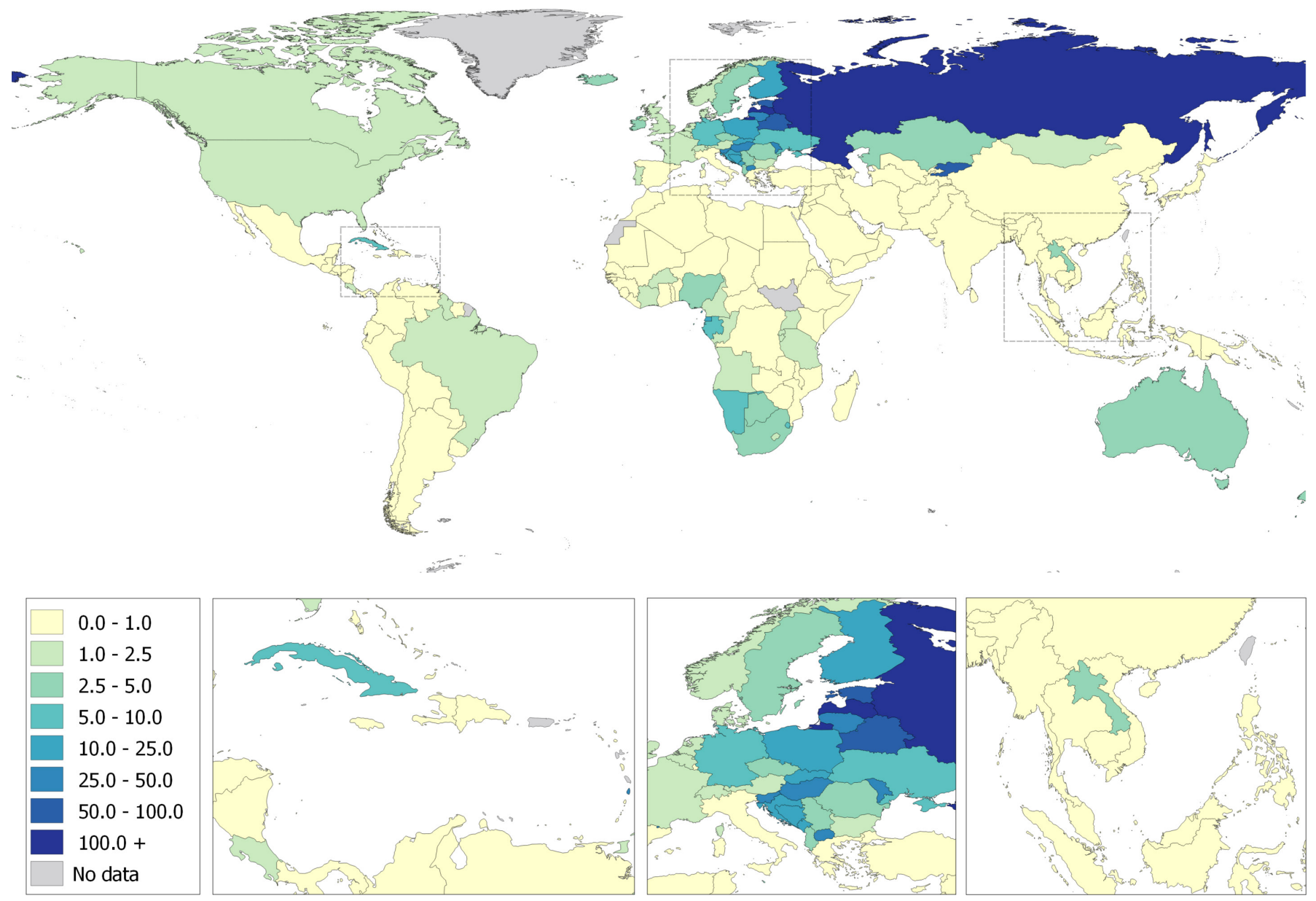

Figure 1 Alcoholic cardiomyopathy mortality rates (deaths per 1000000 adult population) in 2015. Mortality rates denote the number of deaths per 1000000 adult population.

countries, more than $10 \%$ of all cardiomyopathy deaths were caused by alcohol. On the regional level, AAFs above $10 \%$ were found only in Eastern Europe. The largest AAF was found in Kyrgyzstan (67.9\%). Figure 2 illustrates the distribution of AAFs across all countries. As AAF variation was largest in European and Central Asian countries, these regions are additionally highlighted in figure 3 .

For a complete list of ACM deaths, mortality rates and AAFs by sex and country, see online supplementary table B.

\section{Additional analysis}

Comparing civil registration mortality data with predicted fatalities from our models, indications for under-reporting (ie, recorded deaths were lower than predicted lower limits) were identified for 44 out of 91 countries (women: 33; men: 42; for full results see online supplementary table C). In total, 2505 deaths were potentially under-reported in the identified geographies. Under-reported fatalities were concentrated on few countries $(43.1 \%$ of potentially under-reported deaths from Romania, Serbia, France).

In online supplementary table D, ACM deaths from vital statistics or respectively modelled data (main findings of this study) are contrasted with estimates from the GBD study for each country. For the vast majority of countries $(n=172$ out of 190), a higher number of ACM fatalities were reported in the GBD study (median difference $=21$ deaths). Globally, there were
55465 more deaths according to GBD estimates as compared with the findings produced from civil registration data alone.

\section{DISCUSSION \\ Mortality pattern}

There are two major findings that should be highlighted. First, about every 15 th death from cardiomyopathy around the globe was estimated to be attributable to alcohol consumption in 2015 . This estimate is in line with previous estimates ranging between $3 \%$ and $40 \% .^{16}$ Second, the burden of ACM is heavily concentrated on a few countries from Eastern Europe and Central Asia. While in these countries $22.0 \%$ of all global cardiomyopathy deaths were found, they accounted for $82.0 \%$ of all global ACM deaths.

The presented mortality pattern is generally reflecting the global pattern in alcohol-attributable disease burden, ${ }^{15}$ but the described variability between high-burden and low-burden countries appears to be much greater for ACM mortality than for most other alcohol-attributable causes of deaths. One important explanatory factor may be related to different levels and patterns of alcohol consumption impacting on various diseases. ${ }^{17}$ While ACM incidence has been mainly linked to very heavy alcohol consumption over an extended period of time, ${ }^{3}$ much lower levels of ethanol intake are associated with increased risk of various cancers, liver cirrhosis and hypertension. ${ }^{17}$ Thus, a considerable number of alcohol-attributable deaths can be observed around 
Heart failure and cardiomyopathies

Table 2 Regional and global distribution of alcohol-attributable fractions by sex

\begin{tabular}{|c|c|c|c|}
\hline \multirow[b]{2}{*}{ Region } & \multicolumn{3}{|c|}{ Alcohol-attributable fraction } \\
\hline & Total, \% $(95 \% \mathrm{Cl})$ & Female, $\%(95 \% \mathrm{CI})$ & Male, $\%(95 \% \mathrm{CI})$ \\
\hline Andean Latin America & $0.1(0.0$ to 0.9$)$ & $0.1(0.1$ to 0.9$)$ & $0.1(0.0$ to 0.8$)$ \\
\hline Australasia & 4.4 (2.6 to 7.5$)$ & $1.6(0.1$ to 4.9$)$ & 6.2 (4.2 to 9.2 ) \\
\hline Caribbean & $4.3(3.9$ to 5.9$)$ & $0.6(0.4$ to 2.5$)$ & $7.2(6.8$ to 8.6$)$ \\
\hline Central Asia & $8.8(8.2$ to 9.9$)$ & 4.7 (4.4 to 5.4$)$ & 10.9 (10.1 to 12.2$)$ \\
\hline Central Europe & $2.4(1.2$ to 6.4$)$ & $0.8(0.3$ to 3.5$)$ & $4.3(2.5$ to 9.6$)$ \\
\hline Central Latin America & $0.9(0.7$ to 1.8$)$ & 0.2 (0.0 to 0.8$)$ & 1.5 (1.2 to 2.5$)$ \\
\hline Central Sub-Saharan Africa & 1.1 (0.6 to 2.1$)$ & 0.7 (0.3 to 1.6$)$ & 1.7 (1.0 to 2.7$)$ \\
\hline East Asia & $0.6(0.3$ to 1.6$)$ & 0.1 (0.0 to 0.5$)$ & $1.3(0.6$ to 2.8$)$ \\
\hline Eastern Europe & $24.1(17.2$ to 42.9$)$ & $16.6(14.3$ to 27.1$)$ & 28.0 (18.7 to 51.3$)$ \\
\hline Eastern Sub-Saharan Africa & $1.3(0.9$ to 2.1$)$ & $0.4(0.2$ to 0.9$)$ & 2.1 (1.4 to 3.1) \\
\hline High-income Asia Pacific & $0.5(0.01$ to 0.7$)$ & $0.0(0.0$ to 0.9$)$ & $1.0(0.2$ to 2.6$)$ \\
\hline High-income North America & $1.8(0.7$ to 4.4$)$ & 0.7 (0.0 to 2.6$)$ & $2.5(1.1$ to 5.7$)$ \\
\hline North Africa and Middle East & $0.1(0.0$ to 0.4$)$ & $0.1(0.0$ to 0.4$)$ & $0.2(0.1$ to 0.5$)$ \\
\hline Oceania & $0.5(0.2$ to 1.5$)$ & $0.5(0.1$ to 1.9$)$ & $0.4(0.2$ to 1.1$)$ \\
\hline South Asia & $0.4(0.2$ to 1.1$)$ & 0.1 (0.0 to 0.3$)$ & $0.8(0.4$ to 1.7$)$ \\
\hline Southeast Asia & $0.3(0.2$ to 0.9$)$ & 0.2 (0.1 to 0.5$)$ & $0.6(0.3$ to 1.2$)$ \\
\hline Southern Latin America & $0.3(0.1$ to 2.0$)$ & $0.0(0.0$ to 1.3$)$ & 0.7 (0.2 to 2.8$)$ \\
\hline Southern Sub-Saharan Africa & $2.5(1.5$ to 4.2$)$ & $0.6(0.3$ to 1.3$)$ & $4.2(2.6$ to 6.7$)$ \\
\hline Tropical Latin America & $1.4(0.6$ to 3.1$)$ & $0.3(0.0$ to 0.9$)$ & 2.2 (1.0 to 4.6$)$ \\
\hline Western Europe & $2.7(0.7$ to 7.9$)$ & $0.9(0.1$ to 6.2$)$ & 4.1 (1.4 to 9.3$)$ \\
\hline Western Sub-Saharan Africa & 4.1 (2.4 to 7.5$)$ & $0.7(0.3$ to 2.0$)$ & 7.1 (4.1 to 12.3$)$ \\
\hline Global & $6.3(4.2$ to 11.9$)$ & $3.1(2.4$ to 6.0$)$ & $9.0(5.7$ to 16.7$)$ \\
\hline
\end{tabular}

Alcohol-attributable fraction denotes the proportion of alcoholic cardiomyopathy deaths among all deaths due to cardiomyopathy, myocarditis or endocarditis. Definition of regions are based on the Global Burden of Disease study.11

the world, even in countries where alcohol consumption levels are relatively low. ${ }^{15}$ However, as high levels of alcohol use are rare in most countries, few ACM fatalities in these countries are not surprising.

Conversely, the heavy aggregation of ACM deaths in Eastern European can partially be explained by high drinking levels in this region. Traditionally, alcohol use in these countries was higher than elsewhere in Europe or globally, with very heavy binge occasions characterising the local drinking culture. ${ }^{18} 19$ Accordingly, the high ACM mortality burden in Eastern Europe can be regarded as the result of a unique combination of detrimental drinking patterns and alcohol exposure in a wide range of adults during the past two decades. However, decreasing alcohol consumption in these countries and an overall harmonisation of

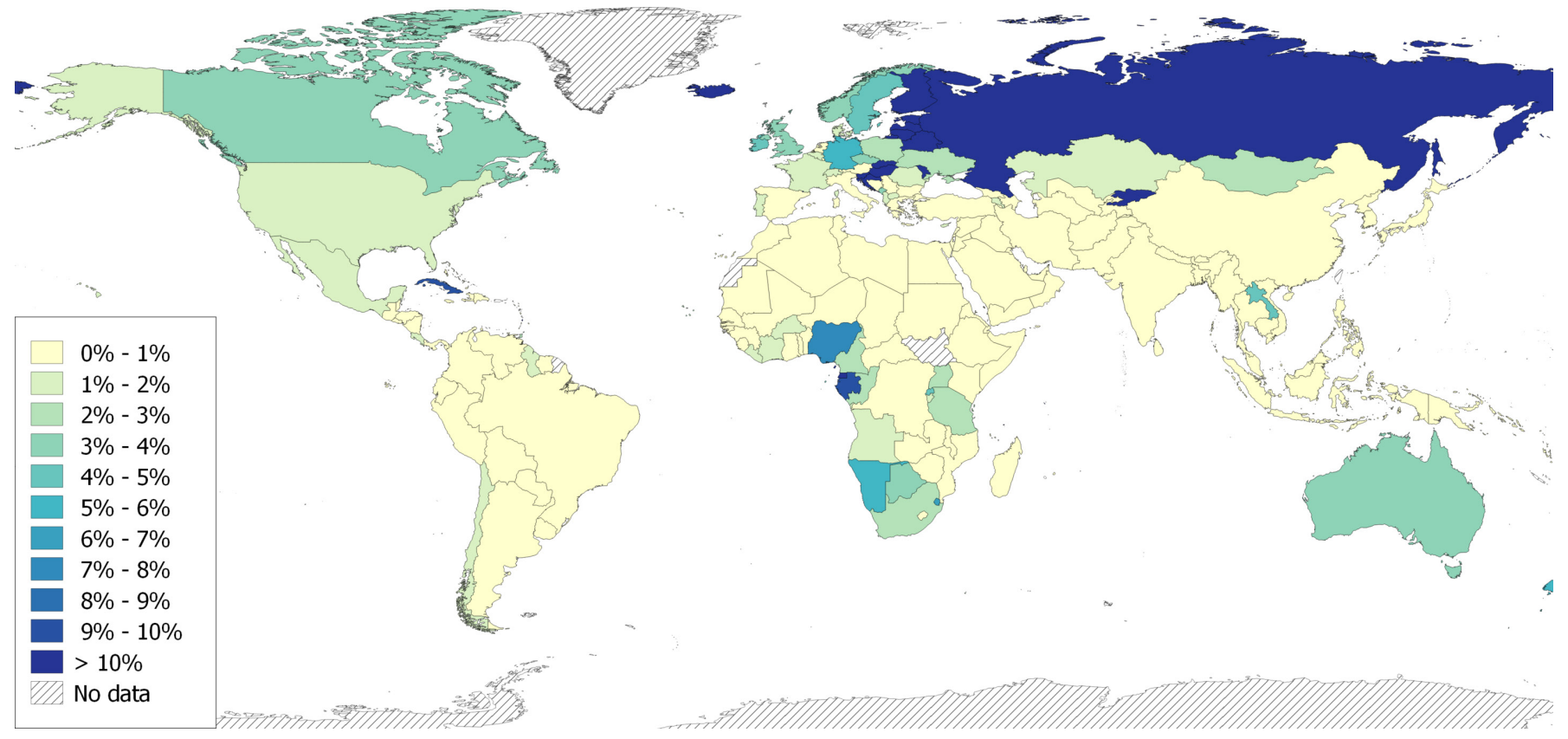

Figure 2 Alcohol-attributable fractions of cardiomyopathy deaths for the total adult population in 2015. Alcohol-attributable fraction denotes the proportion of alcoholic cardiomyopathy deaths among all deaths due to cardiomyopathy, myocarditis or endocarditis. 


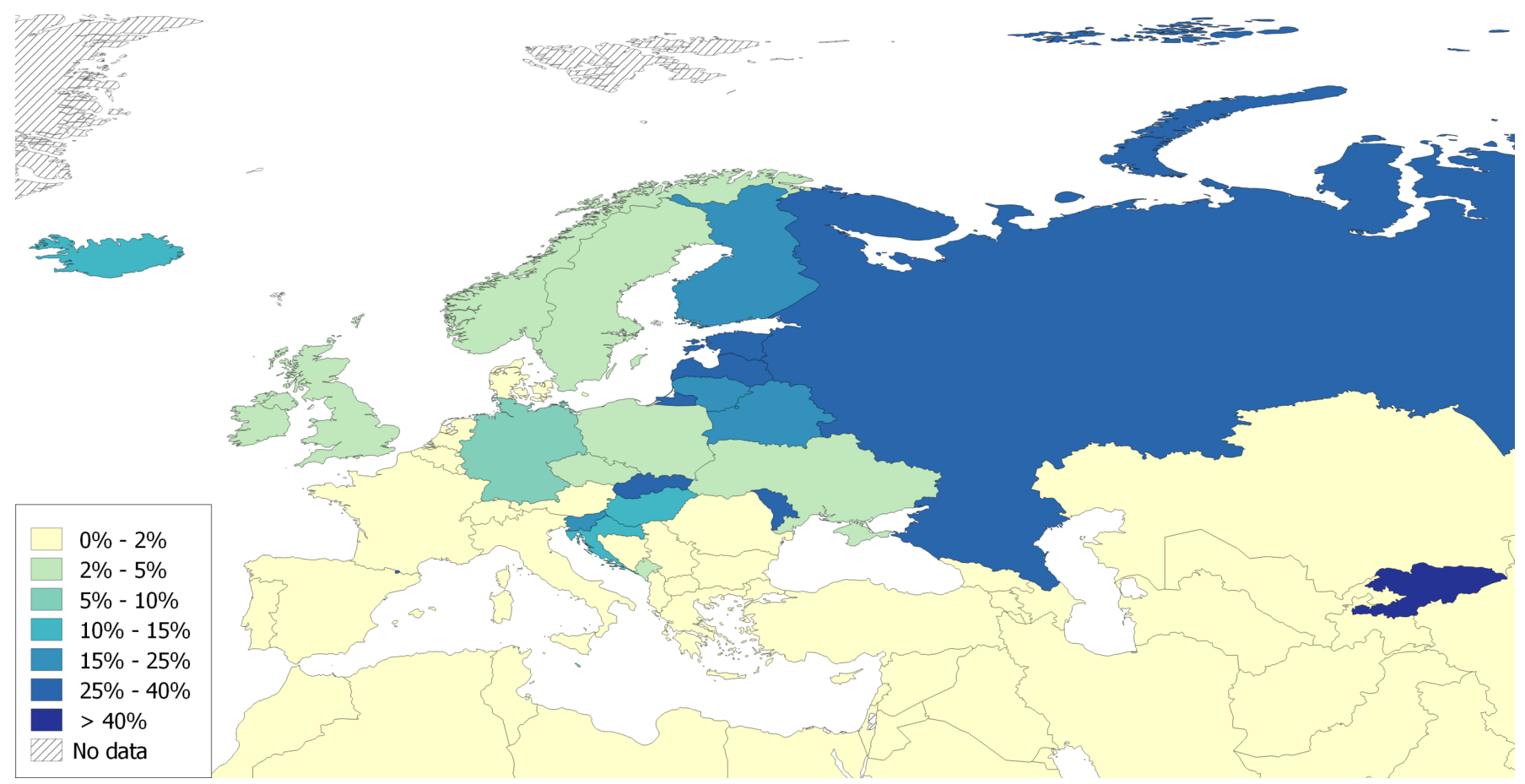

Figure 3 Alcohol-attributable fractions of cardiomyopathy deaths for the total adult population in 2015 for selected countries in Europe and Central Asia. Alcohol-attributable fraction denotes the proportion of alcoholic cardiomyopathy deaths among all deaths due to cardiomyopathy, myocarditis or endocarditis.

drinking patterns in Europe as observed in the past few years ${ }^{18}$ should result in a reduction of ACM deaths in this region in the coming years.

There is another unique feature to Eastern European countries relevant to explain the excessive ACM fatalities in this region. In some former Soviet countries including Belarus, Kyrgyzstan, Russia and Ukraine, autopsies have been legally required for deceased persons under a certain age threshold. ${ }^{10}$ As autopsies are vital for recognising cardiomyopathy (described in detail below) and form an important step in classification of ACM as cause of death, detection rate of ACM might be above average in these countries. Taken together, the observed mortality gap between Eastern European countries and the remaining European region could be attributable to higher alcohol consumption and better detection.

\section{Implications of mortality under-reporting}

There are good reasons to assume that the presented figures are likely to represent only the lower end of the actual mortality burden related to ACM. Most importantly, the presented results are only based on registered deaths, for which the cause of death was unequivocally determined to be ACM. However, misclassification of ACM deaths is likely as cause of death ascertainment can be impaired in various ways. Briefly, there are two strong prerequisites to classifying ACM as the main cause of death: (1) identification of dilated cardiomyopathy, and (2) knowledge of the deceased persons' drinking habit over a lengthy period of time, which allows to attribute the dilation to excessive alcohol use.

With regard to the first requirement, identifying dilated cardiomyopathy as the main cause of death is not an ordinary task. Here, two scenarios can be thought of cases with a cardiomyopathy diagnosis preceding death and cases with a sudden cardiac death (ie, no lifetime cardiomyopathy diagnosis). Dilated cardiomyopathy in live patients can be indicated by rather unspecific symptoms, such as abdominal pain, fatigue or nausea, but formal diagnosis gets only confirmed via echocardiography, MRI and/ or histological analyses. ${ }^{20}$ For individuals who died from a sudden cardiac death, an analogue diagnostic workup may not be feasible. First, sudden cardiac death might not be identified as such (due to drownings, traffic fatalities, etc). Second, imaging techniques may be established as a viable alternative in the future ${ }^{21}$ but autopsy remains the gold standard in identifying the underlying cause of death to date. However, autopsies as well as imaging techniques are far from being routinely applied to deceased persons in most countries, due to lack of resources or ethical considerations.

As outlined, there are numerous barriers in identifying dilated cardiomyopathy as the main cause of death but none of the required diagnostics can determine its specific aetiology. In fact, the second prerequisite to classify ACM implies that the patients' drinking habit needs to be reviewed as one possible cause for dilated cardiomyopathy. While the guidelines of the American Heart Association specify 'a significant history of alcohol use', 22 there is no unambiguous cut-off for a diagnostic decision. ${ }^{3}$ This uncertainty, in addition to the severe stigma associated with alcohol use disorders, ${ }^{23}$ may deter physicians from enquiring into the patients' alcohol use in detail. For deceased persons, acquiring the required information is even more challenging as physicians would need to consult their kin and/or review medical files for an indication of heavy alcohol use (eg, addiction ward admission).

As outlined, there are possible errors for ascertainment of ACM as cause of death, but studies on other alcohol-attributable diseases indicate that misclassification is a systematic phenomenon. ${ }^{12}{ }^{24}$ As information collected during management of previous diseases are often used to issue death certificates, possible diagnostic errors can be carried forward and thus contribute to false cause of death classifications. In fact, cause of death ascertainment in patients with ACM is particularly 
challenging as multiple competing diseases may need to be considered because affected patients are at high risk for other potentially fatal conditions due to their high drinking levels. ${ }^{17}$ In a US study, chronic alcohol use was often omitted from death certificate when other natural diseases were present in the deceased person. ${ }^{24}$

While these are good reasons to assume that ACM deaths are under-reported in vital statistics, there is also empirical support for this claim. First, the GBD study resulted in nearly three times more global ACM deaths as compared with the presented results in this study by accounting for misclassifications in vital statistics. However, the GBD estimates cannot account for all potential misclassification errors outlined above, that is, the true extent of ACM mortality might be even greater.

Second, there are large variations between countries regarding civil registration, which cannot be explained by alcohol exposure. Based on the models in this study, indications for under-reporting could be found for nearly half of countries with vital statistics. For example, Hungary and Serbia are very similar in terms of alcohol exposure and population size, but the gap in ACM deaths is inexplicably wide (Hungary: 221 deaths, Serbia: 20 deaths). Thus, ACM misclassification is likely a greater problem in countries identified as low-end outliers in our models.

\section{Clinical implications}

The extent of misclassification of ACM deaths suggests that this condition is not only underdiagnosed but also undertreated. The presented figures illustrate where interventions are urgently needed. As ACM is preceded by very high drinking levels over several years, ${ }^{3}$ strategies should aim at keeping drinking levels low. One efficient way would be through alcohol screening and subsequent interventions, which was advocated to be implemented routinely in primary healthcare. ${ }^{25}$ As high drinking levels associated with ACM incidence also imply increased risk for incidence of other conditions, ${ }^{17}$ it is plausible to assume that individuals at risk for ACM seek help in various healthcare settings. This is supported by European studies indicating that people with alcohol use disorders are over-represented in primary care settings as compared with the general population, ${ }^{26}$ and high-risk drinkers are more prone to be admitted to in-patient and emergency services than light drinkers. ${ }^{27}$

The quantification of mortality rates can also be of use for facilitating the diagnostic decision-making process. If dilated cardiomyopathy has been diagnosed, it is essential to ascertain the specific cause in order to provide the best treatment. This is especially relevant for ACM as reduction of their drinking levels or complete abstinence is the best way to increase survival rates. $^{22}$ According to recommendations from the American Heart Association, ${ }^{22}$ information from various sources need to be gathered to rule out potential aetiologies. The ideal workup is reasonably complex and involves a lot of data synthesis, which could cause physicians to resort to non-analytical, intuitive heuristic approaches instead. ${ }^{28} 29$ However, these heuristics are overly impacted by cognitive errors, availability and confirmation bias, and they often lead to premature diagnostic decisions and thus to diagnostic errors. ${ }^{29}$ These errors have been identified by comparing diagnoses with autopsy reviews yielding misclassification rates between $10 \%$ and $15 \%$ in various medical fields, ${ }^{28}$ while much higher rates have been reported for alcohol-attributable diseases. ${ }^{12} 24$ There are numerous reasons for diagnostic errors (for a review, see van den Berge and Mamede ${ }^{29}$ ), but knowledge gaps resulting in underestimating the impact of alcohol consumption on various diseases may be responsible for insufficient management of alcohol. These knowledge gaps could be closed by informing physicians about the quantified impact of alcohol on cardiomyopathies. Presenting country-specific and sex-specific attributable fractions of alcohol and other risk factors for dilated cardiomyopathy in future guidelines could help fostering analytical reasoning and balancing the diagnostic workup. This measure is particularly important in countries with large degree of ACM under-reporting.

\section{CONCLUSION}

This study provides a comprehensive assessment of ACM mortality burden, which is heavily concentrated on Eastern European countries. However, vital statistics death counts are likely underestimates with two out of three ACM fatalities being misclassified. The results imply that the condition is currently underdiagnosed and undertreated, which adds to previous claims to implement routine screening and interventions to reduce alcohol-attributable mortality burden. An improved ACM management can contribute to decrease diagnostic errors and to provide the best available treatment.

\section{Limitations}

The interpretation of the results should consider the degree of uncertainty of the input data sources. Specifically, bias in both cardiomyopathy and ACM mortality data cannot be excluded due to great variations in availability and quality of mortality data across countries. ${ }^{9}$ ACM mortality data were mainly available from high-income countries, and no data were available from Sub-Saharan countries. As under-reporting may constitute a major bias in the recorded ACM deaths, the presented figures should be considered lower bound estimates. It should be acknowledged that differences in the age structure of the underlying populations are not reflected in the presented mortality rates, which can introduce a bias in regional comparisons using these figures. Technically, a more extensive modelling strategy with sex-specific and age-group-specific models would have

\section{Key messages}

What is already known on this subject?

- Cardiomyopathy is one of the most common causes of heart failure and resulting death. Alcohol is a major risk factor for this condition.

What might this study add?

- Mortality rates of alcoholic cardiomyopathy vary considerably between regions/geographies, with highest rates in Eastern Europe and Central Asia. While the results corroborate a marked impact of alcohol, the presented estimates should be considered as lower bound estimates due to systematic misclassification of alcoholic cardiomyopathy as a cause of death.

How might this impact on clinical practice?

- Identification of heavy alcohol use and respective interventions should be improved across various healthcare settings. In particular, routine alcohol management in primary healthcare could help to increase detection and subsequent treatment of alcoholic cardiomyopathy. In addition, assessment of and interventions for heavy drinking over time and alcohol use disorders should be integrated into the management of cardiomyopathies. 
allowed age standardisation of the mortality rates. However, such models would have required age-specific alcohol exposure indicators (ie, prevalence of alcohol use disorders) data, which are hardly available for most low-income and middle-income countries, and estimation would have introduced another element of uncertainty. These models would also need to consider that incidence of ACM in a given age group depends on alcohol exposure in the previous age group, due to the considerable time lag between heavy drinking over time and development of ACM. ${ }^{3}$ Importantly, the main focus of this contribution was not to compare mortality rates across locations but to quantify the country-specific and region-specific mortality burden. As these study objectives could be well met with results from sex-stratified models, we decided to perform sex-specific models only.

Contributors JM had full access to all of the data in the study and take responsibility for the integrity of the data and accuracy of the data analysis. Study concept and design: JM and JR. Analysis and interpretation of data: JM. Drafting of the manuscript: JM. Critical revision of the manuscript for important intellectual content: JM, CP, MR and JR. Statistical analysis: JM. Study supervision: JR.

Funding The authors have not declared a specific grant for this research from any funding agency in the public, commercial or not-for-profit sectors.

Competing interests None declared.

Patient consent Not required.

Provenance and peer review Not commissioned; externally peer reviewed.

Data sharing statement All data used in this study are publicly available.

(c) Article author(s) (or their employer(s) unless otherwise stated in the text of the article) 2018. All rights reserved. No commercial use is permitted unless otherwise expressly granted.

\section{REFERENCES}

1 Richardson P, McKenna W, Bristow M, et al. Report of the 1995 World Health Organization/International Society and Federation of Cardiology Task Force on the Definition and Classification of cardiomyopathies. Circulation 1996;93:841-2.

2 World Health Organization. Global Health Estimates. Geneva: World Health Organization, 2017.

3 Rehm J, Hasan OSM, Imtiaz S, et al. Quantifying the contribution of alcohol to cardiomyopathy: A systematic review. Alcohol 2017;61:9-15.

4 Rubin E. Alcoholic myopathy in heart and skeletal muscle. N Eng/ J Med 1979:301:28-33.

5 Urbano-Marquez A, Estruch R, Navarro-Lopez F, et al. The effects of alcoholism on skeletal and cardiac muscle. N Eng/ J Med 1989;320:409-15.

6 Song SK, Rubin E. Ethanol produces muscle damage in human volunteers. Science 1972;175:327-8.

7 Bollinger O. Über die Häufigkeit und Ursachen der idiopathischen Herzhypertrophie in München. Deu Med Wochenschr 1884;10:180-1.
8 Klatsky AL. Alcohol and cardiovascular diseases: where do we stand today? J Intern Med 2015;278:238-50.

9 Mikkelsen L, Phillips DE, AbouZahr C, et al. A global assessment of civil registration and vital statistics systems: monitoring data quality and progress. Lancet 2015;386:1395-406.

10 Manthey J, Imtiaz S, Neufeld M, et al. Quantifying the global contribution of alcohol consumption to cardiomyopathy. Popul Health Metr 2017;15:15.

11 Naghavi M, Abajobir AA, Abbafati C, et al. Global, regional, and national age-sex specific mortality for 264 causes of death, 1980-2016: a systematic analysis for the Global Burden of Disease Study 2016. Lancet 2017;390:1151-210.

12 Pollock DA, Boyle CA, DeStefano F, et al. Underreporting of alcohol-related mortality on death certificates of young US Army veterans. JAMA 1987:258:345-8.

13 World Health Organisation. WHO Mortality Database. Geneva: World Health Organisation, 2017

14 World Health Organisation. Global Information System on Alcohol and Health (GISAH). Geneva: World Health Organisation, 2017.

15 World Health Organization. Global status report on alcohol and health. Geneva: WHO Press, 2014.

16 Taylor MR, Carniel E, Mestroni L. Cardiomyopathy, familial dilated. Orphanet J Rare Dis 2006;1:27

17 Rehm J, Gmel GE, Gmel G, et al. The relationship between different dimensions of alcohol use and the burden of disease-an update. Addiction 2017;112:968-1001.

18 Shield KD, Rylett M, Rehm J. Public health successes and missed opportunities. Trends in alcohol consumption and attributable mortality in the WHO European Region, 1990-2014. Copenhagen, Denmark: WHO European Region, 2016.

19 Popova S, Rehm J, Patra J, et al. Comparing alcohol consumption in central and eastern Europe to other European countries. Alcohol Alcohol 2007;42:465-73.

20 Weintraub RG, Semsarian C, Macdonald P, et al. Dilated cardiomyopathy. The Lancet 2017:390:400-14.

21 Blokker BM, Wagensveld IM, Weustink AC, et al. Non-invasive or minimally invasive autopsy compared to conventional autopsy of suspected natural deaths in adults: a systematic review. Eur Radiol 2016;26:1159-79.

22 Bozkurt B, Colvin M, Cook J, et al. Current diagnostic and treatment strategies for specific dilated cardiomyopathies: a scientific statement from the american heart association. Circulation 2016;134:e579-e646.

23 Schomerus $\mathrm{G}$, Lucht $\mathrm{M}$, Holzinger $\mathrm{A}$, et al. The stigma of alcohol dependence compared with other mental disorders: a review of population studies. Alcohol Alcohol 2011:46:105-12.

24 Daula M, Hanzlick R. Evaluation of certifier practices regarding alcohol-related deaths: Fulton County Medical Examiner's Center, Atlanta, Georgia, 2004. Am J Forensic Med Pathol 2006;27:355-8.

25 OECD. Tackling Harmful Alcohol Use. Sassi F, ed. Economics and Public Health Policy. France: OECD Publishing, 2015

26 Manthey J, Gual A, Jakubczyk A, et al. Alcohol use disorders in Europe: A comparison of general population and primary health care prevalence rates. J Subst Use 2016:21:478-84

27 Miquel L, Manthey J, Rehm J, et al. Risky alcohol use: The impact on health service use in Catalonia. European Addiction Research. submitted.

28 Berner ES, Graber ML. Overconfidence as a cause of diagnostic error in medicine. Am J Med 2008;121:S2-S23.

29 van den Berge K, Mamede S. Cognitive diagnostic error in internal medicine. Eur J Intern Med 2013:24:525-9. 